\section{Hipoglicemia hiperinsulinêmica da infância. Análise de dados clínicos de uma amostra brasileira}

\author{
Hyperinsulinemic hypoglycemia of the infancy. Analysis \\ of clinical data from a Brazilian sample
}

Raphael Del Roio Liberatore Junior', Alcinda Aranha Negri², Carlos Eduardo Martinelli Junior ${ }^{3}$, Cristiane Kochi ${ }^{4}$, Ivani Novato Silva ${ }^{5}$, Paulo Ferrez Collett-Solberg ${ }^{6}$

\section{RESUMO}

${ }^{1}$ Faculdade de Medicina de São José do Rio Preto (Famerp), São José do Rio Preto, SP, Brasil

${ }^{2}$ Faculdade de Medicina da Pontifícia Universidade Católica de São Paulo (PUCSP), São Paulo, SP, Brasil

${ }^{3}$ Faculdade de Medicina de Ribeirão Preto da Universidade de São Paulo (FMRP-USP), Ribeirão Preto, SP, Brasil

${ }^{4}$ Faculdade de Medicina da Irmandade da Santa Casa de Misericórdia de São Paulo (ISCMSP), São Paulo, SP, Brasil

${ }^{5}$ Faculdade de Medicina

da Universidade Federal de Minas Gerais (UFMG), Belo Horizonte, MG, Brasil ${ }^{6}$ Instituto Estadual de Diabetes e Endocrinologia Luiz Capriglione (IEDE), Rio de Janeiro, RJ, Brasil

Correspondência para:

Raphael Del Roio Liberatore Junior

Rua Ondina, 54

15015-205 - São José do Rio Preto

SP, Brasil

liberat@famerp.br

Recebido em 2/Maio/2011

Aceito em 16/Out/2012
Objetivo: Rever a apresentação dos casos de hipoglicemia hiperinsulinêmica da infância (HHI), tratamento e histologia nos serviços de endocrinologia pediátrica no Brasil. Materiais e método: Os serviços receberam protocolo para resgatar dados de nascimento, resultados laboratoriais, tipo de tratamento instituído, necessidade de pancreatectomia e histologia. Resultados: Vinte e cinco casos de $\mathrm{HHI}$ de seis centros foram resgatados, 15 do sexo masculino, 3/25 nascidos de parto normal. A mediana de idade do diagnóstico foi 10,3 dias. As dosagens de glicose e insulina na amostra sérica crítica apresentaram mediana de $24,7 \mathrm{mg} / \mathrm{dL}$ e $26,3 \mathrm{Ul} / \mathrm{dL}$. A velocidade de infusão de glicose endovenosa foi superior a $10 \mathrm{mg} / \mathrm{kg} / \mathrm{min}$ em todos os casos (M:19,1). Diazóxido foi utilizado em 15/25, octreotide em 10 , corticoide em 8 , hormônio de crescimento em 3, nifedipina em 2 e glucagon em 1. Quarenta por cento (10/25) foram pancreatectomizados, nos quais a análise histológica revelou a forma difusa da patologia. Conclusão: Primeira análise crítica de uma amostra brasileira de portadores de HHI congênita. Arq Bras Endocrinol Metab. 2012;56(9):666-71

\section{Descritores}

Hipoglicemia, congênito; hipoglicemia hiperinsulinêmica persistente do lactente; hiperinsulinismo; nesidioblastose

\begin{abstract}
Objective: To review the presentation of hyperinsulinemic hypoglycemia of the infancy (HHI), its treatment and histology in Brazilian pediatric endocrinology sections. Materials and method: The protocol analyzed data of birth, laboratory results, treatment, surgery, and pancreas histology. Results: Twenty-five cases of $\mathrm{HHI}$ from six centers were analyzed: 15 male, 3/25 born by vaginal delivery. The average age at diagnosis was 10.3 days. Glucose and insulin levels in the critical sample showed an average of $24.7 \mathrm{mg} / \mathrm{dL}$ and $26.3 \mathrm{Ul} / \mathrm{dL}$. Intravenous infusion of the glucose was greater than $10 \mathrm{mg} / \mathrm{kg} / \mathrm{min}$ in all cases (M:19,1). Diazoxide was used in 15/25 of the cases, octreotide in 10, glucocorticoid in 8, growth hormone in 3, nifedipine in 2 and glucagon in 1. Ten of the cases underwent pancreatectomy and histology results showed the diffuse form of disease. Conclusion: This is the first critic review of a Brazilian sample with congenital HHI. Arq Bras Endocrinol Metab. 2012;56(9):666-71
\end{abstract}

Keywords

Hypoglycemia, congenital; hyperinsulinemic hypoglycemia of the infancy; hyperinsulinism; nesidioblastosis

\section{INTRODUÇÃO}

A manutenção de adequados níveis glicêmicos é de vital importância em todas as idades, mas é particularmente importante no período neonatal e lactância. Nesse período marcado por intensa atividade metabólica, o cérebro do neonato se torna bastante sensível às reduções glicêmicas, que podem ocasionar danos cerebrais graves (1-4).

A secreção de inapropriados níveis de insulina e sem relação com os níveis de glicemia caracteriza hiperinsulinemia, principal causa de hipoglicemia persistente e recorrente nessa fase da vida. Quando iniciada no 
período neonatal e mantida posteriormente, é denominada forma congênita $(1,5,6)$.

Essa situação clínica, denominada hipoglicemia hiperinsulinêmica da infância forma congênita (HHIC), é marcada pelo aumento descontrolado na secreção de insulina pelas células B pancreáticas, provocando maior utilização da glicose e inibição da produção endógena de glicose, bloqueando a glicólise e a neoglicogênese. Em decorrência desse bloqueio, não ocorre produção de corpos cetônicos $(6)$.

Com exceção de poucos relatos de casos clínicos, não se encontrou publicação de casuística nacional envolvendo HHI. A falta de dados brasileiros e a intenção de implementar a pesquisa molecular desses casos em nosso meio nos estimularam a conhecer as características de amostra multicêntrica brasileira de portadores de HHI ainda vivos.

\section{CASUÍSTICA E MÉTODOS}

Foi encaminhada mensagem eletrônica a todos os serviços de endocrinologia pediátrica do Brasil a partir da lista obtida na Sociedade Brasileira de Pediatria, convidando-os a participarem desse projeto de pesquisa. Responderam a essa solicitação os estados de Mato Grosso, Goiás, Mato Grosso do Sul, Bahia, Sergipe, Alagoas, Pernambuco, Paraíba, Rio Grande do Norte, Ceará, São Paulo, Minas Gerais, Rio de Janeiro, Paraná e Santa Catarina, em um total de 22 serviços. A todos que concordaram em participar, foram encaminhadas cópia do protocolo de pesquisa, aprovação do Comitê de Ética em Pesquisa (CEP) e planilha eletrônica padrão para revisão dos dados. Participaram os serviços de endocrinologia pediátrica da Faculdade de Medicina de Sorocaba, Faculdade de Medicina de São José do Rio Preto, Faculdade de Medicina de Ribeirão Preto da Universidade de São Paulo, Faculdade de Medicina da Universidade Federal de Minas Gerais, Faculdade de Medicina da Irmandade da Santa Casa de Misericórdia de São Paulo e Instituto de Diabetes e Endocrinologia Luiz Capriglione.

Foram resgatados de prontuários médicos os dados: sexo, idade de início, peso e comprimento de nascimento, resultados laboratoriais, forma histológica, tipo de tratamento medicamentoso e realização de pancreatectomia, nos casos ainda vivos de portadores de HHI.

Os dados foram resgatados entre junho de $2010 \mathrm{e}$ fevereiro de 2011 e armazenados em planilha eletrônica Excell para posterior análise em conjunto, com cálculo de parâmetros estatísticos básicos.
O protocolo de pesquisa foi aprovado pelo CEP da Faculdade de Medicina de São José do Rio Preto e foi dispensada a aplicação de Termo de Consentimento.

\section{RESULTADOS}

Foram resgatados os dados de 25 prontuários médicos de portadores de HHI em seguimento atual em seis centros universitários de endocrinologia pediátrica.

Desses 25 casos, 15 são do sexo masculino, três dessas crianças nasceram de parto normal e as demais (22), de parto operatório, com idades gestacionais entre 33 semanas e 6 dias a 39 semanas, mediana de 38 semanas e 1 dia. Os dados antropométricos de nascimento são mostrados na tabela 1 .

A idade do diagnóstico de hipoglicemia variou de 1 a 240 dias, sendo que, em 4 dos 25 casos, o diagnóstico da hipoglicemia foi feito em período superior a 90 dias. A mediana de idade do diagnóstico da hipoglicemia, sem esses quatro casos, foi de 10,3 dias.

A suspeita clínica de hipoglicemia foi feita a partir da presença de apatia e hipotonia em todos os casos.

Tabela 1. Dados antropométricos e tipo de parto de 21 dos casos de HHI

\begin{tabular}{|c|c|c|c|c|}
\hline Caso & $\begin{array}{l}\text { Tipo de } \\
\text { parto }\end{array}$ & $\begin{array}{c}\text { Peso } \\
\text { (gramas) }\end{array}$ & $\begin{array}{c}\text { Comprimento } \\
\text { (cm) }\end{array}$ & $\begin{array}{c}\text { Idade } \\
\text { gestacional } \\
\text { (semanas) }\end{array}$ \\
\hline 1 & C & 5.250 & 55 & 38 \\
\hline 2 & C & 4.570 & 54 & 38 \\
\hline 3 & C & 2.500 & 45 & 36 \\
\hline 4 & C & 2.390 & & 34 \\
\hline 5 & C & 1.615 & & 33 \\
\hline 7 & C & 2.500 & 47 & 38 \\
\hline 8 & C & 3.870 & & 39 \\
\hline 9 & C & 3.400 & 48 & 38 \\
\hline 11 & $\mathrm{~N}$ & 3.700 & 49 & 38 \\
\hline 12 & C & 3.850 & & 38 \\
\hline 13 & C & 3.650 & 49,5 & 38 \\
\hline 14 & C & 3.050 & & 38 \\
\hline 15 & C & 4.300 & 51 & 38 \\
\hline 16 & C & 2.570 & 49 & 38 \\
\hline 17 & $\mathrm{~N}$ & 3.440 & 51 & 39 \\
\hline 18 & NR & 4.520 & 54 & 39 \\
\hline 19 & $\mathrm{~N}$ & 3.440 & 50,5 & 38 \\
\hline 20 & C & 2.075 & 44 & 38 \\
\hline 21 & C & 2.950 & 48,5 & 39 \\
\hline 22 & C & 3.840 & & 39 \\
\hline 23 & C & 3.310 & 49 & 39 \\
\hline
\end{tabular}

C: cesária; N: normal; NR: não resgatado. 
Em 20 dos casos, ocorreram também taquicardia e hipotermia. Em um dos casos, exatamente o que mais tempo demandou para o diagnóstico, crise convulsiva era o diagnóstico inicial.

A dosagem de glicose no momento da coleta da amostra crítica variou de 6 a $48 \mathrm{mg} / \mathrm{dL}$, com mediana de $24,7 \mathrm{mg} / \mathrm{dL}$. A velocidade de infusão de glicose foi maior que $10 \mathrm{mg} / \mathrm{kg} / \mathrm{min}$ em todos os casos, com mediana de 19,1. A dosagem de insulina sérica à amostra crítica variou de 7 a $147 \mathrm{mUI} / \mathrm{mL}$, mediana de 26,3.

A dosagem de cetonemia não foi realizada em nenhum dos casos, amônia sérica em sete, ácido úrico em três e lactato sérico em dez dos casos. Os resultados laboratoriais são mostrados na tabela 2 .

Com relação ao tratamento, diazóxido foi utilizado em 15 dos 25 casos, octreotide em dez, corticoide em

Tabela 2. Resultados de amostras laboratoriais sanguíneas de portadores de HHI (as dosagens de cetonas, amônia, lactato e ácido úrico estão sem unidades)

\begin{tabular}{|c|c|c|c|c|c|c|}
\hline Caso & $\begin{array}{c}\text { Glicemia } \\
\text { mg/dl }\end{array}$ & $\begin{array}{l}\text { Insulina } \\
\mathrm{mUI} / \mathrm{ml}\end{array}$ & $\begin{array}{l}\text { Cetona } \\
\mathrm{mmol} / \mathrm{l}\end{array}$ & $\begin{array}{c}\text { Amônia } \\
\text { mg/l }\end{array}$ & $\begin{array}{l}\text { Lactato } \\
\mathrm{mmol} / \mathrm{l}\end{array}$ & $\begin{array}{c}\text { Ácido } \\
\text { úrico } \\
\mathrm{mg} / 100 \mathrm{ml}\end{array}$ \\
\hline 1 & 25 & 32,5 & NR & & $\mathrm{NR}$ & NR \\
\hline 2 & 20 & 18 & $\mathrm{NR}$ & $\mathrm{NR}$ & $\mathrm{NR}$ & NR \\
\hline 3 & 23 & 11 & 0 & NR & 2 & NR \\
\hline 4 & 15 & 82 & 0 & 559 & 7,3 & NR \\
\hline 5 & 23 & 20 & 0 & NR & 5,4 & NR \\
\hline 6 & 31 & 25 & 0 & NR & 2,3 & NR \\
\hline 7 & 14 & 7 & 0 & NR & 6,8 & 10,8 \\
\hline 8 & 6 & 32 & 0 & NR & 4,4 & 7,8 \\
\hline 9 & 48 & 3 & NR & 30,3 & 2,4 & NR \\
\hline 10 & 23 & 147 & NR & NR & $\mathrm{NR}$ & NR \\
\hline 11 & 20 & 13,5 & NR & NR & B & NR \\
\hline 12 & 35 & 8,66 & $\mathrm{NR}$ & NR & NR & NR \\
\hline 13 & 12 & 8 & $\mathrm{NR}$ & $\mathrm{NR}$ & $\mathrm{NR}$ & NR \\
\hline 14 & 23 & 7 & 46 & NR & $\mathrm{NR}$ & NR \\
\hline 15 & 20 & 12 & 39 & NR & NR & NR \\
\hline 16 & 36 & 7,1 & 0 & 127 & 7,88 & NR \\
\hline 17 & 32 & 4,1 & 0 & NR & NR & NR \\
\hline 18 & 32 & 4,3 & 0 & 14 & 1,9 & NR \\
\hline 19 & 13 & 38,2 & 0 & NR & 3,6 & 2 \\
\hline 20 & 31 & 6,5 & NR & 14 & NR & NR \\
\hline 21 & 31 & $\mathrm{NR}$ & NR & 72 & $\mathrm{NR}$ & NR \\
\hline 22 & 26 & NR & $\mathrm{NR}$ & NR & NR & NR \\
\hline 23 & 19 & 17 & NR & NR & NR & NR \\
\hline 24 & 25 & 40 & NR & NR & NR & NR \\
\hline 25 & 15 & 7 & NR & NR & NR & NR \\
\hline
\end{tabular}

NR: não realizado. oito, hormônio de crescimento em três, nifedipina em dois e glucagon em um desses 25 casos. As doses utilizadas estão mostradas na tabela 3 .

Tabela 3. Medicamentos e doses utilizadas nos portadores de HHI

\begin{tabular}{|c|c|c|c|c|c|c|c|}
\hline Caso & $\begin{array}{c}\text { GH } \\
\text { U/kg/ } \\
\text { dia }\end{array}$ & $\begin{array}{c}\text { Cort } \\
\mathrm{MG} / \mathrm{m}^{2}\end{array}$ & $\begin{array}{l}\text { Gluc } \\
\text { Ug/kg/ } \\
\text { min }\end{array}$ & $\begin{array}{c}\text { Octreot } \\
\text { ug/kg/d }\end{array}$ & $\begin{array}{c}\text { Diazóxido } \\
\text { mg/kg/d }\end{array}$ & $\begin{array}{c}\text { Cirur- } \\
\text { gia }\end{array}$ & $\begin{array}{c}\text { Nife } \\
\mathrm{mg} / \mathrm{kg} / \mathrm{d}\end{array}$ \\
\hline 1 & & 20 & & 40 & 50 & & \\
\hline 2 & & & & & 15 & & \\
\hline 3 & & & & & 7,5 & & \\
\hline 4 & 0,2 & 20 & & & & Sim & \\
\hline 5 & & 20 & & & 15 & & \\
\hline 6 & & 20 & & & & Sim & \\
\hline 7 & & & & & 15 & & \\
\hline 8 & 0,1 & 35 & & 30 & & Sim & \\
\hline 9 & & & & & & & $1,5 / \mathrm{kg} / \mathrm{d}$ \\
\hline 10 & 0,1 & 20 & 15 & 10 & & Sim & \\
\hline 11 & & 15 & & 15 & & Sim & \\
\hline 12 & & & & 3 & 10 & & $1,0 / \mathrm{kg} / \mathrm{d}$ \\
\hline 13 & & & & & & Sim & \\
\hline 14 & & 25 & & & & Sim & \\
\hline 15 & & & & 250 & 10 & Sim & \\
\hline 16 & & & & 20 & 10 & & \\
\hline 17 & & & & 20 & & & \\
\hline 18 & & & & 20 & & & \\
\hline 19 & & & & 20 & 15 & & \\
\hline 20 & & & & & 12 & & \\
\hline 21 & & & & & 15 & Sim & \\
\hline 22 & & & & & 15 & & \\
\hline 23 & & & & & 10 & & \\
\hline 24 & & & & & 8 & Sim & \\
\hline 25 & & & & & 15 & & \\
\hline
\end{tabular}

GH: hormônio de crescimento; Cort: hidrocortisona; Gluc: glucagon; Nife: nifedipina.

A associação do uso de corticoide e octreotide foi utilizada em três casos e corticoide e hormônio de crescimento também em três casos. Diazóxido e octreotide foram utilizados em cinco casos.

Em 10 dos 25 casos, ocorreu falha do tratamento medicamentoso com necessidade de pancreatectomia. Dos 14 casos em que houve a utilização do diazóxido, em três a resposta ao tratamento foi falha e esses casos necessitaram de pancreatectomia.

Em todos os casos que sofreram pancreatectomia, a descrição histológica foi da forma difusa da doença pancreática. 


\section{DISCUSSÃO}

Várias são as causas da HHI. Essas podem ser congênitas, secundárias, associadas a síndromes genéticas ou associadas a doenças metabólicas $(1,2)$.

As formas secundárias são normalmente transitórias e se associam a presença de diabetes materno, restrição de crescimento intrauterino, asfixia ou incompatibilidade Rh. Várias síndromes genéticas podem estar associadas à $\mathrm{HH}$, tais como Beckwith-Wiedemann, Soto, Trissomia do 13, Turner mosaico e outras. As desordens congênitas da glicosilação $(\mathrm{CDG})$ e a tirosinemia tipo 1 são as doenças metabólicas que cursam com HHI (3-6).

As formas congênitas de HHI constituem um grupo bastante heterogêneo de condições e são as formas mais graves e mais frequentes de HHI (2).

Essas formas de HHI congênitas (HHC) podem se apresentar com variadas características clínicas e subgrupos histológicos, mas o aspecto mais recentemente estudado é o das diferenças moleculares.

Os sinais e sintomas de hipoglicemia são os marcadores clínicos da $\mathrm{HH}$ e podem ocorrer em qualquer faixa etária.

No período neonatal, esses sinais e sintomas são inespecíficos e a possibilidade de hipoglicemia deve ser sempre descartada na vigência de palidez, má-perfusão tecidual, letargia, falta de sucção, convulsões (2).

A rapidez da suspeita diagnóstica e consequente rápida instalação da conduta adequada é de fundamental importância para reduzir a morbidade, bastante elevada nessa doença. Na casuística apresentada, o tempo médio para diagnóstico foi bastante elevado, 10 dias. Mais preocupante ainda foi a detecção tardia, que ocorreu em praticamente $20 \%$ da casuística (quatro casos). Séries da literatura mostram o mesmo retardo no diagnóstico e especulam se esta não seria a causa da morbidade elevada $(6,7,8)$.

Vale ainda a pena ressaltar que os sinais e sintomas de hipoglicemia em recém-nascidos são bastante inespecíficos e que hipoglicemia deve ser aventada toda vez em que ocorra mudança de padrão clínico.

Macrossomia como resultado dos níveis elevados de insulina intrauterinamente pode ocorrer como sinal clínico, embora macrossomia não ocorra em todos os casos. Na casuística apresentada, macrossomia ocorreu em quatro casos.

A necessidade de infusão contínua de glicose em níveis maiores do que $10 \mathrm{mg} / \mathrm{kg} /$ minuto para a manutenção dos níveis glicêmicos e a dependência do aporte de glicose endovenosa são marcadores de $\mathrm{HH}$.
Na casuística apresentada, houve a necessidade de grandes aportes de glicose por via endovenosa em todos os casos. Causa surpresa a necessidade de aportes muito altos e provavelmente se relaciona à demora na instalação de tratamento medicamentoso adequado $(7,8)$.

Durante o episódio de hipoglicemia, que pode ser espontâneo ou provocado pelo jejum, a confirmação diagnóstica da $\mathrm{HH}$ necessita da coleta de exames adicionais conhecidos como "amostra crítica" pela maioria dos autores $(7,8)$.

A amostra crítica é a coleta de amostras para dosagem sérica de glicose, cetonas, ácidos graxos, gasometria ( $\mathrm{pH}$ e bicarbonato), lactato, piruvato, amônia, hormônio de crescimento, cortisol e insulina e/ou peptídeo $\mathrm{C}$, além da pesquisa para erros inatos de metabolismo. Em alguns serviços, há também a realização do teste de estímulo de glucagon.

O diagnóstico laboratorial de $\mathrm{HH}$ envolve a presença de hipoglicemia com presença de nível dosável de insulina, cetonemia e dosagem de ácidos graxos negativos. Por vezes, somente uma dosagem não é suficiente para a conclusão diagnóstica e, na dúvida, a necessidade de altas taxas de infusão endovenosa de glicose deve dirigir o diagnóstico.

$\mathrm{Na}$ casuística apresentada, foi realizada a dosagem de glicose e insulina em todos os casos. O restante das dosagens do que os autores denominam amostra crítica foi muito pouco realizado e de forma pouco padronizada. A dosagem de cetonemia pode ser realizada pela utilização de fitas reagentes em aparelhos que dosam também glicemia capilar. A dosagem de amônia, lactato e cetonemia não está disponível em todos os serviços e mesmo os resultados dos nossos casos não puderam ser comparados pela grande disparidade entre os resultados. Esses exames são importantes, pois algumas formas de HHI se associam a alterações desses exames, que permitem orientar quanto ao tratamento e à ocorrência em outras gestações.

Com relação à fisiopatologia, a HHI pode envolver respectivamente defeitos nos canais de potássio das células B pancreáticas ou acúmulo de metabólitos intermediários que desencadeiam a secreção de insulina (9-12).

Nas células B pancreáticas, os canais de potássio sensíveis à adenosina-trifosfato (canal KATP) exercem papel fundamental no controle da síntese de insulina estimulada pela glicose. Esses canais são complexos protéicos heterooctaméricos transmembrana e compostos de 4 canais retificadores de potássio (Kir6.2) e 4 receptores 1 de sulfonilureia (SURl). 
Quando a glicose se acopla ao canal KATP, leva ao aumento da relação ATP/ADP intracelular e fechamento desses canais, com consequente despolarização da membrana celular, influxo de cálcio e exostose da insulina.

Defeitos desses canais podem resultar em secreção deficiente ou, no caso da HHI, secreção descontrolada de insulina $(13,14)$.

A droga de escolha para o tratamento da HHI é o diazóxido. Essa medicação funciona abrindo os canais KATP e suprimindo a liberação de insulina pela célula $\beta$. É considerada a droga de escolha pela possibilidade de uso por via oral (15), com exceção nos casos como mutação nos canais KATP.

A utilização de glucagon e octreotide por via subcutânea contínua constitui a forma de terapia de segunda linha, especificamente naqueles casos em que não ocorre resposta ao diazóxido. Esses dois medicamentos permitem ainda o uso crônico e prolongado em nível ambulatorial (16-18).

$\mathrm{Na}$ casuística estudada, foi observado que a utilização de diazóxido ocorreu em pouco mais da metade dos casos e a dificuldade de obtenção da droga com certeza contribuiu para esse reduzido uso. Os glicocorticoides foram amplamente utilizados e ainda hoje a maioria dos serviços de neonatologia indica a utilização desse medicamento para os casos de hipoglicemia neonatal, entretanto, seus efeitos colaterais e a possibilidade do uso do diazóxido fazem com que os glicocorticoides possam ser deixados de lado. Essa utilização, principalmente antes da coleta da amostra crítica, dificulta muito também a análise etiológica dos casos. Glucagon foi surpreendentemente utilizado em apenas um dos casos.

$\mathrm{Na}$ associação de medicamentos, observaram-se o mesmo viés do hábito da utilização dos glicocorticoides e a baixa utilização do glucagon, de custo bem mais reduzido que o octreotide.

Duas formas histológicas se apresentam nos casos de $\mathrm{HH}$, com aumento no volume celular das células B pancreáticas, representando uma maior atividade biológica e conhecida como adenomatose pancreática.

A forma difusa, conhecida como nesidioblastose, apresenta todas as células $\mathrm{B}$ com o padrão histológico anteriormente descrito. Pode ser familiar ou esporádica e ser produto de mutação de transmissão recessiva ou dominante.

$\mathrm{Na}$ forma focal, somente pequenas regiões do pâncreas, medindo de 2 a $10 \mathrm{~mm}$, apresentam adenomatose. Esses casos são normalmente esporádicos.
A pancreatectomia é a forma de terapia de escolha para as formas focais de HHI e para os casos difusos que não respondem ao tratamento medicamentoso. Embora a pancreatectomia evolua para insuficiência exócrina e diabetes melito, acaba sendo a única alternativa de tratamento.

Nos casos desta casuística, em dez se fez a opção pela pancreatectomia.

A totalidade dos casos operados se mostrou da forma difusa. Acreditamos que também aqui tenha ocorrido viés, pois não dispomos no país da análise molecular para HHI e de mesma forma não temos serviços capacitados para a realização da localização da lesão focal. Na realização da avaliação histológica, o patologista escolhe a região com maior quantidade de adenomatose $\mathrm{e}$ não examina o restante do pâncreas.

Este é o primeiro relato brasileiro de casuística de portadores de HHI da forma congênita e ainda em seguimento clínico. Trata-se de doença de alta morbimortalidade e não muito frequente. As maiores casuísticas da literatura envolvem a descrição em torno de 100 casos e acreditamos termos, portanto, atingido um número bastante representativo de casos.

Notamos que a pesquisa diagnóstica, sem a adequada coleta da amostra crítica e principalmente o tratamento, pela elevada utilização de glicocorticoides e pequena utilização de diazóxido, necessita ser mais bem padronizada com a participação dos serviços de neonatologia.

Agradecimentos: ao suporte financeiro da Fundação de Apoio ao Ensino, Pesquisa e Assistência (Faepa) do Hospital das Clínicas da Faculdade de Medicina de Ribeirão Preto da Universidade de São Paulo e da Fundação de Apoio à Pesquisa e Extensão de São José do Rio Preto (Faperp).

Declaração: os autores declaram não haver conflitos de interesse científico neste estudo.

\section{REFERÊNCIAS}

1. Stanley CA. Hyperinsulinism in infants and children. Pediatr Clin North Am. 1997;44:363-74.

2. Hussain K, Balnkestein O, De Lonlay P, Christesen HT. Hyperinsulinemic hypoglycaemia: biochemical basis and the importance of maintaining normoglycaemia during management. Arch Dis Child. 2007;92:598-70.

3. Bruninig GJ. Recent advances in hyperinsulinism and the pathogenesis of diabetes mellitus. Curr Opin Pediatr. 1990;2:758-65.

4. Fournet JC, Junien C. The genetics of neonatal hyperinsulinism. Horm Res. 2003;59:30-4.

5. Glaser B, Thornton PS, Otonkoski T, Junien C. The genetics of neonatal hyperinsulinism. Arch Dis Child. 2000;82:79-86. 
6. De Lonlay P, Fournet JC, Touati G, Groos MS, Martin D, Sevin C, et al. Heterogeneity of persistent hyperinsulinaemic hypoglycaemia. A series of 175 cases. Eur J Pediatr. 2002;161:37-48.

7. Meissner T, Mayatepek E. Clinical and genetic heterogeneity in congenital hyperinsulinism. Eur J Pediatr. 2002;161:6-20.

8. Sempoux C, Guiot $Y$, Lefevre A, Nihoul-Fekete C, Jaubert F, Saudubray JM, et al. Neonatal hyperinsulinemic hypoglycemia: heterogeneity of the syndrome and keys for differential diagnosis. $\mathrm{J}$ Clin Endocrinol Metab. 1998;83:1455-61.

9. Marthinet E, Bloc A, Oka Y, Tanizawa Y, Wehrle-Haller B, Bancila V, et al. Severe congenital hyperinsulinism caused by a mutation in the Kir6.2 subunit of the adenosine triphosphate-sensitive potassium channel impairing trafficking and function. J Clin Endocrinol Metab. 2005;90:5401-6.

10. Tornovsky S, Crane A, Cosgrove KE, Hussain K, Lavie J, Heyman $M$, et al. Hyperinsulinism of infancy: novel ABCC8 and KCNJ11 mutations and evidence for additional locus heterogeneity. J Clin Endocrinol Metab. 2004;89:6224-34.

11. Huopio $H$, Reimann F, Ashfield R, Komulainen J, Lenko HL, Rahier $\mathrm{J}$, et al. Dominantly inherited hyperinsulinism caused by a mutation in the sulfonylurea receptor type 1. J Clin Invest. 2000;106:897-906.
12. Pinney SE, MacMullen C, Becker S, Lin YW, Hanna C, Thornton P, et al. Clinical characteristics and biochemical mechanisms of congenital hyperinsulinism associated with dominant KATP channel mutations. J Clin Invest. 2008;118:2877-86.

13. James C, Kapoor RR, Ismail D, Hussain K. The genetic basis of congenital hyperinsulinism. J Med Genet. 2009;46:289-99.

14. Flanagan $S E$, Clauin $S$, Bellanne-Chantelot $C$, Lonlay $P$, Harries $\mathrm{LW}$, Gloyn AL, et al. Update of mutations in the genes encoding the pancreatic beta-cell K(ATP) channel subunits Kir6.2 (KCNJ11) and sulfonylurea receptor 1 (ABCC8) in diabetes mellitus and hyperinsulinism. Human Mutation. 2009;30:170-80.

15. Flanagan SE, Kapoor RR, Mali G, Cody D, Murphy N, Schwahn B, et al. Diazoxide-responsive hyperinsulinemic hypoglycemia caused by HNF4A gene mutations. Eur J Endocrinol. 2010;162:987-92.

16. Hussain K. Diagnosis and management of hyperinsulinaemic hypoglycaemia of infancy. Horm Res. 2008;69:2-13.

17. Kapoor RR, James C, Hussain K. Advances in the diagnosis and management of hyperinsulinemic hypoglycemia. Nat Clin Pract Endocrinol Metab. 2009;5:101-12.

18. Kapoor RR, Flanagan SE, James C, Shield JP, Ellard S, Hussain K. Hyperinsulinaemic hypoglycaemia. Arch Dis Child. 2009;94:450-7. 